\title{
NOVOS FILMES DE NANOCOMPÓSITOS ENTRE ÓXIDO DE GRAFENO REDUZIDO E AZUL DA PRÚSSIA OBTIDOS PELO MÉTODO INTERFACIAL
}

\author{
C. R. SOUZA ${ }^{1}$, C. B. de AQUINO², J. C. PIERETTIE ${ }^{2}$, S. H. DOMINGUES ${ }^{2}$ e E. NOSSOL ${ }^{3}$ \\ ${ }^{1}$ Universidade Federal de Uberlândia, Faculdade de Engenharia Química \\ ${ }^{2}$ Universidade Presbiteriana Mackenzie, Mackgraphe - Graphene and Nano-Material Research \\ Center \\ ${ }^{3}$ Universidade Federal de Uberlândia, Instituto de Química \\ E-mail para contato: enossol@ufu.br
}

\begin{abstract}
RESUMO - Neste trabalho sintetizaram-se materiais nanoestruturados do tipo óxido de grafeno reduzido/azul da Prússia pelo método interfacial, através de soluções bifásicas, no qual se obteve filmes homogêneos, que foram caracterizados por espectroscopia Raman, constatando bandas características de compostos de carbono, com hibridizações $\mathrm{sp}^{2}$ e $\mathrm{sp}^{3}$, além de bandas relacionadas ao azul da Prússia, atestando a formação do material nanocompósito. A atividade redox do azul da Prússia também foi estudada, através da análise dos pares redox presentes nas medidas de voltametria cíclica, com a presença de quatro picos característicos do AP. A análise das imagens de microscopia eletrônica de varredura mostraram uma morfologia esférica das nanopartículas de AP, e que as mesmas estão dispostas entre as folhas de óxido de grafeno reduzido.
\end{abstract}

\section{INTRODUÇÃ̃O}

\subsection{Grafeno}

O grafeno (Figura 1), nome da monocamada de grafite e do mais recente alótropo de carbono, consiste em uma rede bidimensional constituída por uma estrutura hexagonal de átomos de carbono, com hibridização $\mathrm{sp}^{2}$ e espessura monoatômica. Sendo assim, é considerado a unidade básica fundamental que forma as estruturas de outros materiais de carbono, como os fulerenos, nanotubos e fibras de carbono.

Por suas propriedades únicas, o grafeno é considerado mais resistente que o diamante. Assim, podem-se citar propriedades destacadas para esse material, tais como a alta resistência mecânica, elevada transparência e flexibilidade. Além disso, é considerado um semicondutor de band gap zero, pois sua banda de valência "toca" a banda de condução, permitindo que o material tenha propriedades condutoras, mas difere de metais comuns por não possuir elétrons livres na banda de condução, visto isso apresenta certa condutividade elétrica. (Wang et al, 2010).

Um dos métodos de síntese mais utilizados para o grafeno é a esfoliação das folhas de grafite por oxidação com utilização de ácidos e oxidantes fortes, como $\mathrm{HNO}_{3}, \mathrm{KMnO}_{4}, \mathrm{H}_{2} \mathrm{O}_{2}$. 


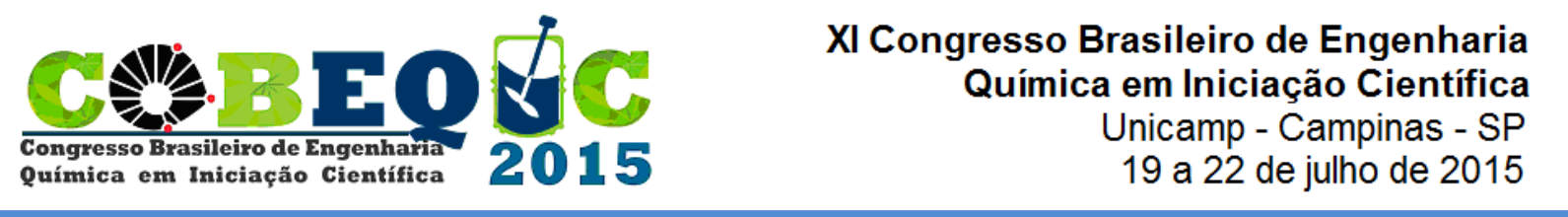

Consequentemente, as folhas de grafeno obtidas apresentam alguns grupos funcionais, como ácidos carboxílicos, hidroxilas, entre outros, e este é denominado óxido de grafeno (GO). Após esse processo oxidativo, a rede $\mathrm{sp}^{2}$ do grafite é parcialmente distorcida, e pode ser restaurada por métodos de redução, no qual ocorre a eliminação de grande parte dos grupos funcionais, e obtêm-se o óxido de grafeno reduzido (rGO), contendo ainda alguns grupos funcionais (Edwards et al, 2013) .

Figura 1 - Representação esquemática do grafeno.

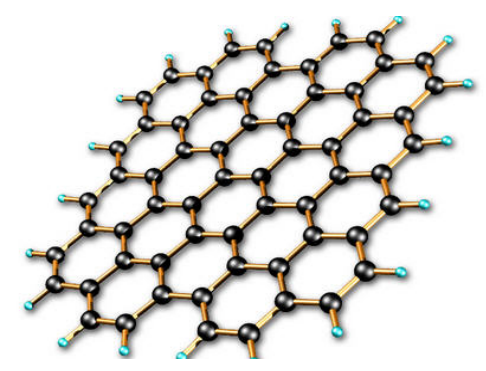

\subsection{Azul da Prússia}

O azul da Prússia (AP) é composto de coordenação de cor azul escura, que tem estrutura representada pela fórmula $\mathrm{Fe}_{4}\left[\mathrm{Fe}(\mathrm{CN})_{6}\right]_{3} \cdot \mathrm{xH}_{2} \mathrm{O} \quad(\mathrm{x}=14-16)$, apresentando uma estrutura cúbica de face centrada, como mostra a figura 2.

III

Duas estruturas distintas para o AP foram propostas, uma apresentando $\mathrm{K}^{+}$, KFe $\mathrm{Fe}^{I I}(C N)_{6}$ (solúvel) e outra apresentando íons $\mathrm{Fe}^{3+}, \mathrm{Fe}_{4}\left[\mathrm{Fe}^{I I}(C N)_{6}\right]$ (insolúvel). Na estrutura chamada insolúvel ocorre a falta de um quarto das unidades $\mathrm{Fe}^{\mathrm{II}}(\mathrm{CN})_{6}$ da cela unitária, a fim de manter a eletroneutralidade. Esse arranjo confere algumas vacâncias na estrutura cristalina que são ocupadas por moléculas de água. Além disso, cada esfera de coordenação de cada centro de $\mathrm{Fe}^{\mathrm{III}}$ ao redor da vacância é preenchida por moléculas de água. (Nossol at al, 2013)

Vale ressaltar que os termos "solúvel" e "insolúvel" utilizados não dizem respeito a uma solubilidade propriamente dita, mas a uma tendência do composto em peptizar (processo de transporte entre fases pelo qual passa um determinado coloide ao se misturar com uma fase dispersiva).

O azul da Prússia, em suas duas disposições estruturais, apresenta baixíssima solubilidade real em água, sendo pouco solúvel para todos os fins práticos, pois seu produto de solubilidade é da ordem de $10^{-40}$. Entretanto, a mesma aumenta em meios fortemente alcalino e ácidos (com pH próximo de 1,0). (Tosta et al, 2008).

Tendo em vista as limitações quanto às aplicações do azul da Prússia devido à estabilidade destes hexacianometalatos, uma das formas mais promissoras de se aumentar essa estabilidade, além de propiciar efeitos sinérgicos no material final, consiste na preparação de nanocompósitos entre o AP e materiais a base de carbono. 
Figura 2 - Cela unitária do azul da Prússia e seus núcleos de ferro coordenados octaedricamente.

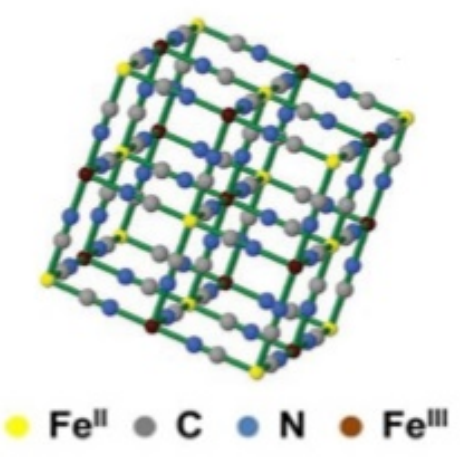

A obtenção de materiais nanocompósitos entre AP e materiais derivados do grafeno, como o rGO, vem propiciando a observação de propriedades maximizadas, como efeitos eletrocatalíticos acentuados e aumento da estabilidade do nanocompósito (Jiang et al, 2011). A preparação desses materiais tem sido realizada através de diferentes métodos (Kumar et al, 2014), esse trabalho utilizará o método interfacial para a preparação do nanomaterial.

\section{OBJETIVOS}

O objetivo desse trabalho é a síntese pelo método interfacial filmes finos transparentes de $\mathrm{rGO} / \mathrm{AP}$, obtendo um compósito estável e com propriedades diferenciadas, definidas pelo efeito sinergético dessa combinação. Além disso, tem-se ainda como objetivo a caracterização desses filmes através de espectroscopia Raman, voltametria cíclica, imagens de microscopia eletrônica de varredura, e posteriormente outros métodos como espectroscopia UV-Vis e difratometria de raios $\mathrm{X}$.

\section{PARTE EXPERIMENTAL}

Como o objetivo é obter um filme fino pelo método interfacial, ou seja, a partir de soluções bifásicas tem-se a formação de uma fina camada de filme, que será retirada em diferentes substratos (ITO, silício, quartzo, PET - polietilenoteraftalato); para isso é necessário a preparação de duas soluções:

i. Fase orgânica: utilizou-se uma dispersão contendo $2,0 \mathrm{mg}$ de $\mathrm{rGO}$ em $20 \mathrm{~mL}$ de tolueno, com auxílio de um banho de ultrassom por cerca de 40 minutos. $\mathrm{FeCl}_{3}(\mathrm{pH} \mathrm{2})$.

ii. Fase aquosa: preparou-se $25 \mathrm{~mL}$ de uma solução $0,1 \times 10^{-3} \mathrm{~mol} \mathrm{~L}^{-1} \mathrm{de}^{K_{3}\left[\mathrm{Fe}(C N)_{6}\right] \mathrm{e}}$

Em seguida foram adicionados $20 \mathrm{~mL}$ da fase aquosa em agitação magnética em um balão de fundo redondo de $50 \mathrm{~mL}$, com posterior adição de $20 \mathrm{~mL}$ da fase orgânica. Após uma hora de agitação magnética foram adicionados $10 \mu \mathrm{L} \mathrm{H}_{2} \mathrm{O}_{2}$ de uma solução 0,5 mol L-1 
de $\mathrm{H}_{2} \mathrm{O}_{2}$. $\mathrm{O}$ sistema permaneceu sobre agitação magnética por 4 horas, obtendo-se um filme azul escuro na interface.

Após o período de reação, a fase aquosa foi lavada com água destilada até atingir um valor de $\mathrm{pH}$ em torno de 7. Para a remoção do filme nos substratos, estes devem estar previamente lavados e presos no PET e em uma haste metálica. Retirou-se o filme formado do balão e adicionou-o, através de uma pipeta, em um béquer contendo água destilada e a haste metálica com os substratos (ITO, silício) fixados. Para recolher o filme sobre os substratos basta erguer a haste metálica até que ela entre em contato com o filme na superfície da água.

Como experimento controle foi feita a síntese de um filme somente de rGO, utilizando-se o mesmo processo descrito anteriormente porém, ao invés da solução de $\mathrm{K}_{3}\left[\mathrm{Fe}(\mathrm{CN})_{6}\right]$ na fase aquosa foi utilizado $20 \mathrm{~mL}$ de água destilada, com agitação magnética durante $5 \mathrm{~h}$ e sem adição de $\mathrm{H}_{2} \mathrm{O}_{2}$.

As medidas de voltametria cíclica foram realizadas em um potenciostato Autolab modelo PGSTAT12 com velocidade de varredura de $50 \mathrm{mV} . \mathrm{s}^{-1}$. Os espectros Raman foram coletados em um espectrofotômetro Renishaw Raman-Image acoplado a um microscópio óptico, o laser utilizado foi de $\operatorname{Ar}(514 \mathrm{~nm})$. As imagens de microscopia eletrônica de varredura foram adquiridas em um equipamento Mira FEG-SEM (TESCAN) em $10 \mathrm{kV}$.

\section{RESULTADOS E DISCUSSÃO}

A solução aquosa foi previamente acidificada, pois como mencionado anteriormente, o azul da Prússia aumenta sua real solubilidade em meio ácido, tornando assim o $\mathrm{pH}$ por volta de 3 .

Além disso, foi adicionado $\mathrm{H}_{2} \mathrm{O}_{2}$ (peróxido de hidrogênio) durante a síntese para que um dos centros de $\mathrm{Fe}^{3+}$, do $\mathrm{K}_{3}\left[\mathrm{Fe}(\mathrm{CN})_{6}\right]$ (ferricianeto de potássio) ou do $\mathrm{FeCl}_{3}$ (cloreto férrico) se reduzisse. Assim seria possível a formação de azul da Prússia, o qual possui um centro de $\mathrm{Fe}^{3+}$ e outro de $\mathrm{Fe}^{2+}$.

As amostras do compósito rGO/AP foram caracterizadas através de espectroscopia Raman. Assim foram obtidos espectros de amostras de filmes de rGO e do compósito rGO/AP, como mostra as Figuras 4-A-a e 4-A-b, respectivamente.

Como é possível observar, na figura 4-A-a, temos duas bandas características de compostos de carbono. A banda $\mathrm{D}$, localizada em aproximadamente $1350 \mathrm{~cm}^{-1}$, associada às vibrações dos átomos de carbono com hibridização $\mathrm{sp}^{3}$, defeitos estruturais, carbonos amorfos e heteroátomos. Também observa-se a banda $\mathrm{G}$, em aproximadamente $1590 \mathrm{~cm}^{-1}$, relacionada ao modo $E_{2 g}$ de vibrações de estiramento simétrico no plano, correspondente aos átomos de carbono $\mathrm{sp}^{2}$. Ainda assim é pode-se observar a banda G', ou 2D, localizada em aproximadamente $2640 \mathrm{~cm}^{-1}$, que representa um grau maior de organização estrutural, e devido às reações químicas no processo de redução do rGO apresenta baixa intensidade. Essa banda não está relacionada com a presença de defeitos (Saito et al., 2013) . 
Figura 4 - Espectros Raman do rGO (A-a) e do nanocompósito rGO/AP (A-b).

Voltamograma cíclico do filme rGO/AP (B).
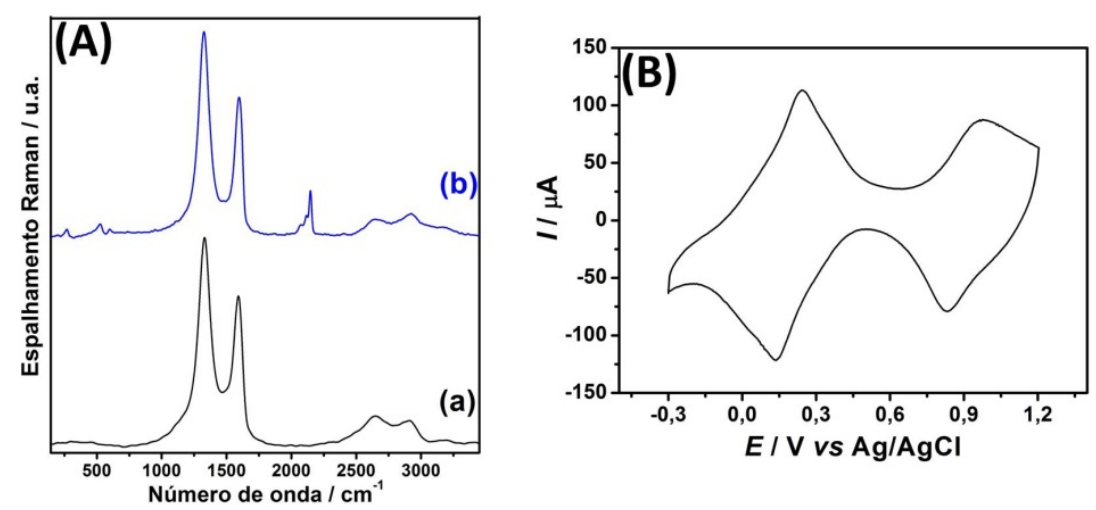

Na Figura 4-A-b, além das bandas características do rGO, D e G, temos bandas características do azul da Prússia, presentes em 530 e em $2150 \mathrm{~cm}^{-1}$. A primeira refere-se ao estiramento $\mathrm{Fe}-\mathrm{CN}$, e a segunda é atribuída às vibrações $\mathrm{C} \equiv \mathrm{N}$ (Mažeikienè et al., 2011).

O filme nanocompósito rGO/AP também foi caracterizado por voltametria cíclica. A Figura 4-B mostra um voltamograma com a presença de dois pares redox, típicos do azul da Prússia, atribuídos aos seguintes processos redox (Itaya et al., 1982) :

$$
\begin{aligned}
& \mathrm{Fe}_{4}{ }^{I I I}\left[\mathrm{Fe}^{I I}(\mathrm{CN})_{6}\right]_{3}+4 e^{-}+4 K^{+} \longrightarrow \mathrm{K}_{4} \mathrm{Fe}_{4}{ }_{4}^{I I}\left[\mathrm{Fe}^{I I}(\mathrm{CN})_{6}\right]_{3} \\
& \mathrm{Fe}_{4}{ }^{I I I}\left[\mathrm{Fe}^{I I}(\mathrm{CN})_{6}\right]_{3}+3 A^{-} \rightleftarrows \mathrm{Fe}_{4}{ }^{I I I}\left[\mathrm{Fe}^{I I I}(\mathrm{CN})_{6} \mathrm{~A}\right]_{3}+3 e^{-}
\end{aligned}
$$

As imagens de microscopia eletrônica de varredura, presentes na Figura 5, mostram uma morfologia rugosa para o filme de rGO (Figura 5-a), as quais cobrem o substrato de maneira homogênea.

Figura 5 - Imagens de MEV do filme de rGO (a) e do nanocompósito rGO/AP (b)
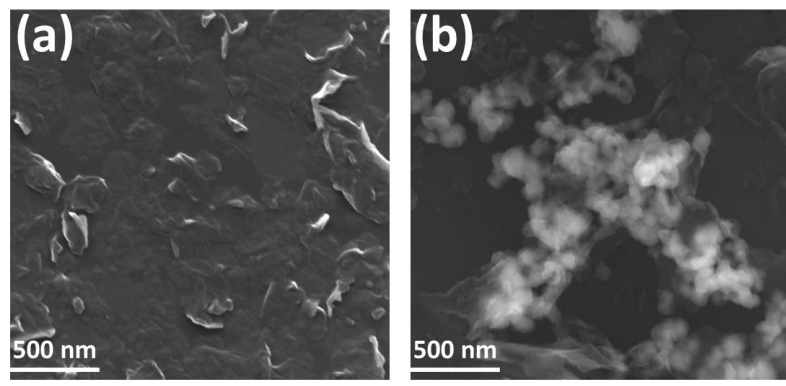

O filme nanocompósito rGO/AP (Figura 5-b) mostra a presença de nanopartículas de AP distribuídas ao longo das folhas de rGO, sendo mais uma evidência do sucesso deste método de síntese para preparação do material.

\section{CONCLUSÕES}


O método interfacial foi utilizado com sucesso para preparação de nanocompósitos do tipo $\mathrm{rGO} / \mathrm{AP}$. As diferentes técnicas de caracterização revelaram a obtenção de um material homogêneo e com atividade redox.

Os resultados revelam a necessidade de caracterizações adicionais com relação às propriedades do compósito. Dessa maneira serão realizados estudos mais específicos visando um detalhamento completo do material. Também será realizado o controle da morfologia das nanopartículas de AP, através de variações nos parâmetros de síntese e consequentemente obtenção de novas propriedades visando diferentes aplicações.

\section{REFERENCIAS}

EDWARDS, R. S., COLEMAN, K. S., Graphene synthesis: relationship to applications. Nanoscale, v. 5, p.38-51, 2013.

ITAYA, K., ATAKA, T., TOSHIMA, S., Spectroelectrochemistry and electrochemical preparation method of Prussian blue modified electrodes. J. Am. Chem. Soc., v. 104, p.4767-4772, 1982

JIANG, Y., ZHANG, X., SHAN, C., HUA, S., ZHANG, Q., BAI, X., DAN, L., NIU, L., Functionalization of graphene with electrodeposited Prussian blue towards amperometric sensing application. Talanta, v. 85, p.76-81, 2011.

KUMAR, A., PETERS, E. C., BURGHARD, M., Self-assembled magnetic nanoparticles of Prussian blue on graphene. RSC Adv., v. 4, p. 18061-18064, 2014.

MAŽEIKIENĖ, R., NIAURA, G., MALINAUSKAS, A., Electrocatalytic reduction of hydrogen peroxide at Prussian blue modified electrode: An in situ Raman spectroelectrochemical study. J. Electroanal. Chem ., v. 660, p.140-146, 2011.

NOSSOL, E. Filmes finos, transparentes e multifuncionais de nanocompósitos entre nanotubos de carbono e azul da Prússia. 2013. 135f. Tese de Doutorado.

SAITO, R., HOFMANN, M., DRESSELHAUS, G., JORIO, A., DRESSELHAUS, M. S., Raman spectroscopy of graphene and carbon nanotubes. Adv. Phys., v. 60, p.413-550, 2011.

TOSTA, M. L. Comportamento eletrocrômico de filmes de azul da Prússia em carbonato de Propileno. 2008. 113f. Dissertação de Mestrado.

WANG, X.-L., DOU, S. X., ZHANG, C., Zero-gap materials for future spintronics, electronics and optics. NPG Asia Mater, v. 2, p.31-38, 2010. 